\title{
SATELLITE IMAGE COMPRESSION ALGORITHM BASED ON THE FFT
}

\author{
Khaled Sahnoun and Noureddine Benabadji \\ University of Sciences and Technology of Oran- Algeria, department of physics \\ Laboratory of Analysis and Application of Radiation
}

\begin{abstract}
Image compression is minimizing the size in bytes of a graphics file without degrading the quality of the image to an unacceptable level, the reduction in file size allows more images to be stored in a given amount of disk or memory space, it also reduces the time required for images to be sent over the ground This paper presents a new coding scheme for satellite images. In this study we apply the fast Fourier transform and the scalar quantization for standard LENA image and satellite image, The results obtained after the (SQ) phase are encoded using entropy encoding, after decompression, the results show that it is possible to achieve higher compression ratios, more than $78 \%$, the results are discussed in the paper.
\end{abstract}

\section{KEYWORDS}

Encoding Entropy, Fast Fourier transform, Compression, $S Q$.

\section{INTRODUCTION}

Satellites image compression is used to minimize the amount of memory needed to represent this image [1], satellites images often require a large number of bits to represent them, and if the image needs to be transmitted to the ground or stored, it is impractical to do so without somehow reducing the number of bits for this data [2][3]. In this study we have developed a technique of satellites image compression based on the FFT "Fourier transform" and SQ "scalar quantization" to compression and decompression Satellites image, with satisfactory quality of the reconstructed image.

\section{FFT}

The Fourier transform (FFT), also known as frequency analysis or spectral involved in the implementation of many digital techniques for processing signals and images [4] It is found in applications such as direct harmonic analysis of musical signals and vibrations, but also reduces the rate coding of speech and music, compression, and digital transmissions Applying a Fourier transform give a complex image [5], we calculated the module Fm and the phase Fp of the original image, and we represent the module. These 02 images can be defined as following:

$$
\begin{aligned}
& \operatorname{Re}(F(f)(x, y))=F m(x, y) \cos (F p(x, y)) \rightarrow(1) \\
& \operatorname{Im}(F(f)(x, y))=F m(x, y) \sin (F p(x, y)) \rightarrow(2)
\end{aligned}
$$


The International Journal of Multimedia \& Its Applications (IJMA) Vol.6, No.1, February 2014

Where Im, Re denotes the imaginary and real parts, one then finds that Fp is not unique in general, to represent the transform, it is only the module

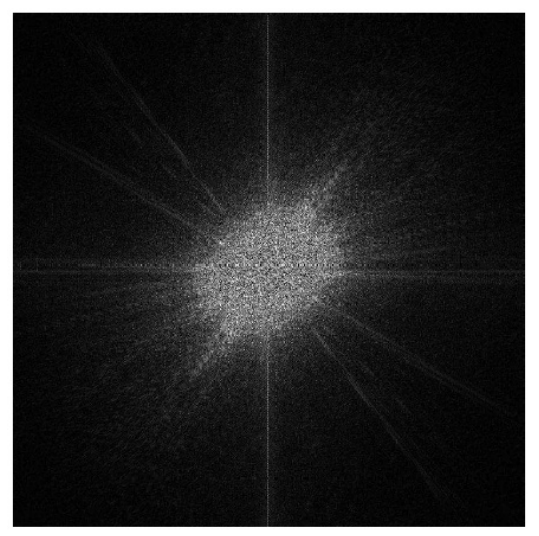

Figure 1. Module blue channel butterfly

The image is square, we completed the butterfly image with black to make it square. In addition, we work more frequently with square image [6]

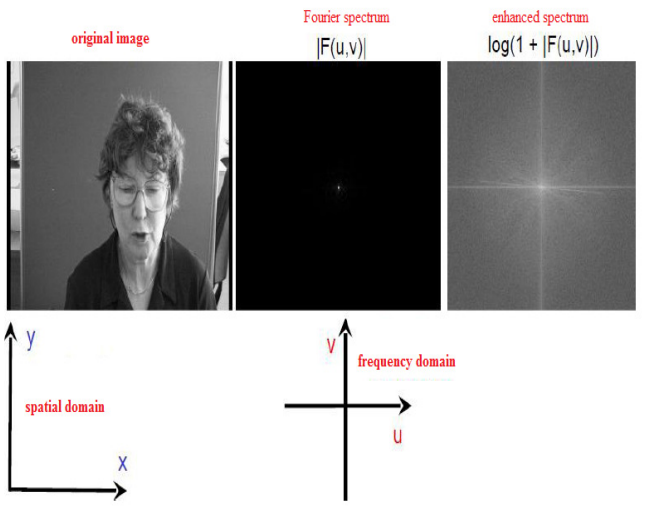

Figure 2. Fourier Transform (FFT).

The FFT of a real image can be expressed as follows

$$
F(u, v)=\frac{1}{m n} \sum_{x=0}^{m-1} \sum_{y=0}^{n-1} f(x, y) \cdot e^{-2 \pi i\left(\frac{u x}{m}+\frac{v y}{n}\right)}
$$

With $\mathrm{u}, \mathrm{v}=0 . . \mathrm{N}-1 \rightarrow(3)$

Reverse:

$$
f(x, y)=\sum_{u=0}^{m-1} \sum_{v=0}^{m-1} F(u, v) \cdot e^{2 \pi i\left(\frac{u x}{m}+\frac{v y}{n}\right)}
$$

With $\mathrm{x}, \mathrm{u}=0 . . \mathrm{N}-1 \rightarrow(4)$ 
The International Journal of Multimedia \& Its Applications (IJMA) Vol.6, No.1, February 2014

The variables $\mathrm{u}, \mathrm{v}$ used in the equation (3) are variable frequency $\mathrm{x}, \mathrm{y}$ used in the equation (4) are variable in the spatial domain, $\mathrm{F}(\mathrm{u}, \mathrm{v})$ Is often represented by its amplitude and phase, the formula is given by:

$$
\begin{gathered}
\operatorname{amplitude}(F(u, v))=\sqrt{R^{2}(u, v)+I^{2}(u, v)} \rightarrow(5) \\
\text { phase }(F(u, v))=\tan ^{-1}\left\lfloor\frac{I(u, v)}{R(u, v)}\right\rfloor \rightarrow(6)
\end{gathered}
$$

\section{PROPOSED APPROACH}

The main philosophies of our image compression technique based on the fast Fourier transform (FFT). The general scheme of the algorithm relied on the steps shown in the following figure

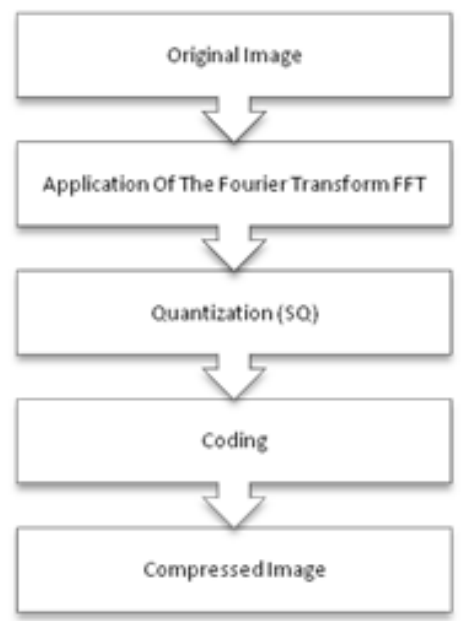

Figure 3. The steps of the proposed algorithm

\subsection{Read the image source}

The input image is satellite picture, the size will be equal to $2^{\mathrm{n}}$, in our case it is $2^{8}$

\subsection{Calculate the FFT}

The data returned by the FFT is complex which contains real and imaginary parts, in this method only the amplitude is concerned, which is the only party represented in the displays of the results.

\subsection{Quantization}

The compression by quantization can be improved either by acting on the step of constructing the dictionary or by acting on the quantization step of the input pixels. In this method, an improvement of the second step has been proposed. The SQ of each is the approximate value of the signal $x(t)$ by a value that belongs to a set of codes $\{y 1, y 2, \ldots ., y l\}$, at any amplitude $x$ in the interval [xi-1, xi], there corresponds a quantized value yi situated in that Interval [8]

\subsection{Coding}

The image coding by R.L.E algorithm coded the sequence of identical gray pixel values, assigning the three parameters the position $(\mathrm{x}, \mathrm{y})$ of the first pixel in the sequence and the gray value of the first pixel, the length of the sequence. The Huffman algorithm is applied finally which is a compression algorithm capable of generating variable length codes to a whole number 
The International Journal of Multimedia \& Its Applications (IJMA) Vol.6, No.1, February 2014

of bits, this algorithm can achieve good results, but it should be kept the codebook used between the compression/decompression algorithm [9]

\section{EXPERIMENTAL RESULTS}

\subsection{Evaluation of compression}

Compression ratio $(\mathrm{T} \%)$ :

$$
\begin{gathered}
\mathrm{Q}=\frac{\text { initial size }}{\text { final size }} \rightarrow(7) \\
\mathrm{T}=\frac{1}{0} \rightarrow(8)
\end{gathered}
$$

Compression gain:

$$
\mathrm{G}=\frac{\text { initial size }- \text { final size }}{\text { final size }} \rightarrow(9)
$$

Mean Squared Error:

$$
\operatorname{MSE}=\frac{1}{N} \sum_{i=0}^{N-1}(n \operatorname{comp}(i)-n(i))^{2} \rightarrow(10)
$$

Peak Signal to Noise Ratio PSNR

$$
P S N R=10 \log _{10} \frac{255^{2}}{M S E} d b \rightarrow(11)
$$

A powerful algorithm has a gain of maximum compression (T) and a minimum mean square error [7] , the compression ratio and MSE error, PSNR are calculated by equations (8), (6) and (11). The proposed algorithm has been tested by Lena picture and satellite image.

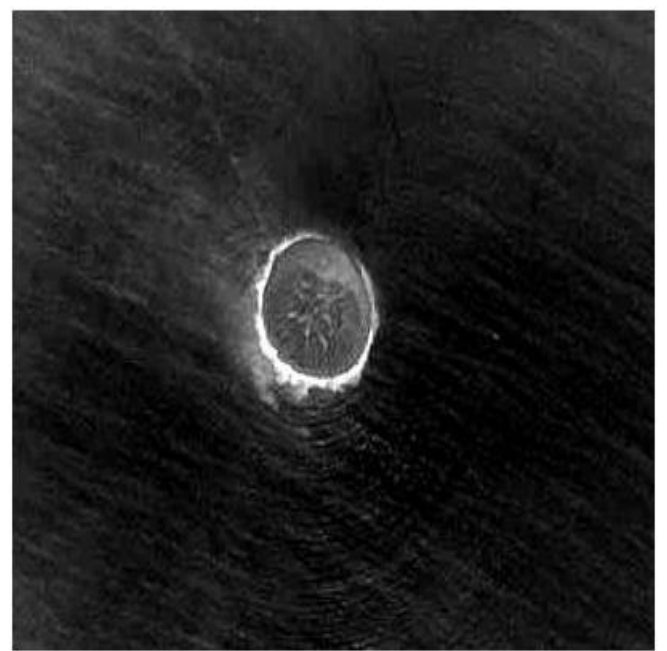

Figure 4. Original satellite image (1) 
The International Journal of Multimedia \& Its Applications (IJMA) Vol.6, No.1, February 2014

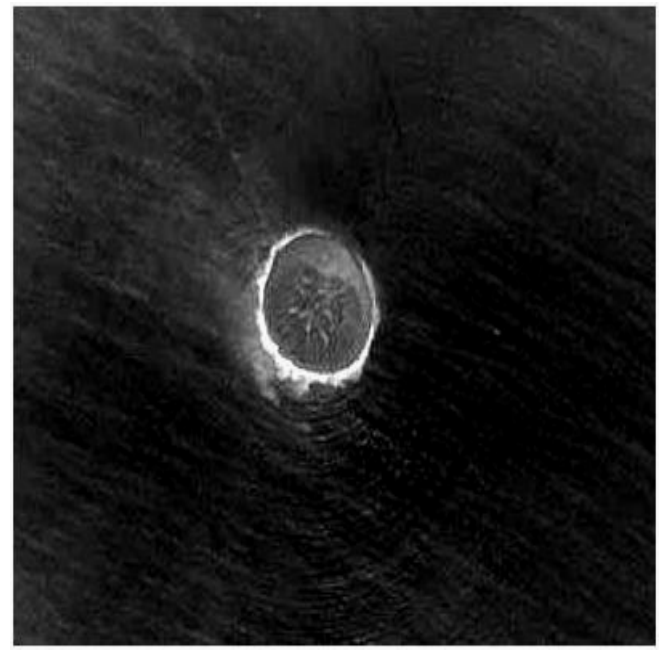

Figure 5. Satellite image reconstructed (2)

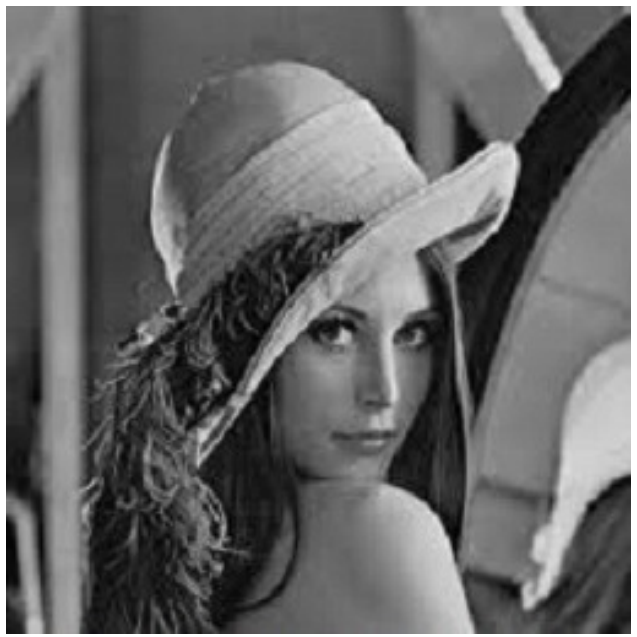

Figure 6. Original Lena image

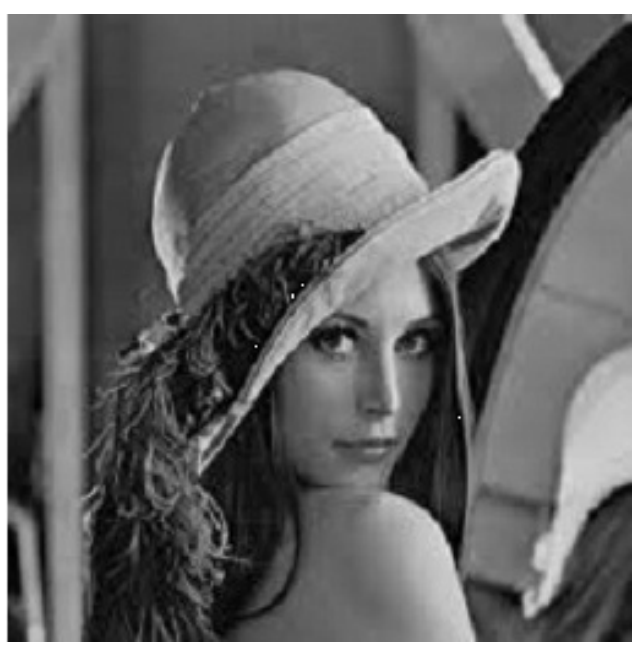

Figure 7. Reconstructed Lena image 
The International Journal of Multimedia \& Its Applications (IJMA) Vol.6, No.1, February 2014

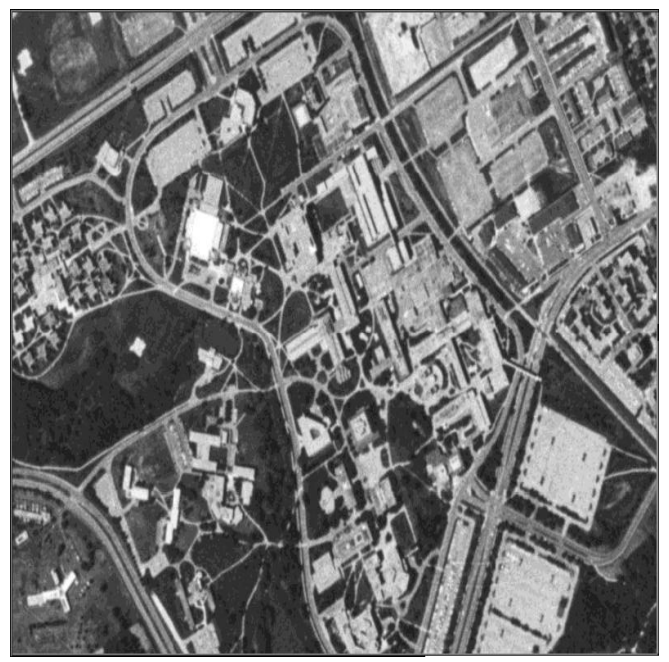

Figure 8. Original satellite image (2)

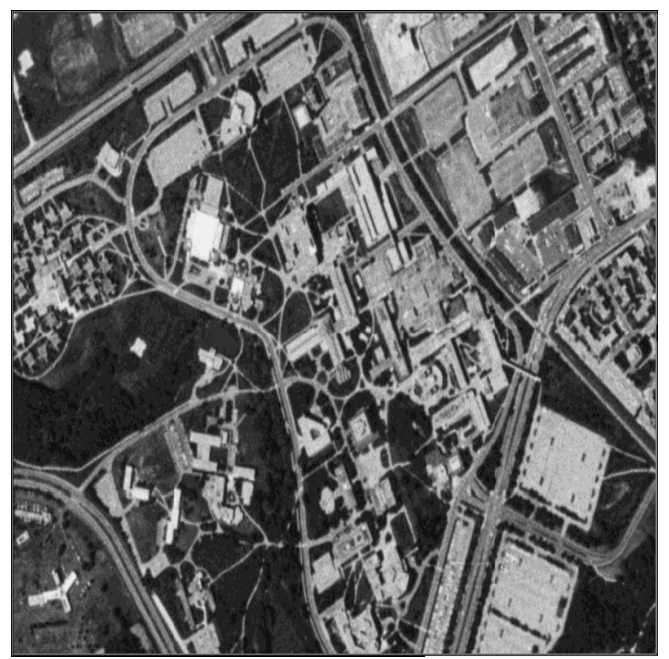

Figure 9. Satellite image reconstructed (2)

Table 1. Table of results with Lena and satellite image.

\begin{tabular}{|c|c|c|c|}
\hline Image & Lena & Satellite (1) & Satellite (1) \\
\hline MSE & 11.67 & 9.92 & 8.87 \\
\hline PSNR (db) & 37.49 & 38.20 & 33.79 \\
\hline T (\%) & 65.52 & 87.27 & $76 . .3$ \\
\hline
\end{tabular}

\section{CONCLUSIONS}

In this paper we have presented an algorithm for satellite image compression based on the Fourier transform (FFT) and the scalar quantization (SQ), the compression/decompression algorithm that we have presented in this study is able to compress image with high quality compression and better quality, tests on Lena imagei show the superiority of this algorithm. 
The International Journal of Multimedia \& Its Applications (IJMA) Vol.6, No.1, February 2014

\section{REFERENCES}

[1] L. MOURAD "new approach for image compression based on wavelet and fractal for Meteosat image application” PhD Thesis University Mouloud Mammeri UMMTO Tizi-Ouzou Algeria 2011

[2] STEVEN PIGEON "contributions to the data compression" PhD Thesis University of Montreal Canada 2001.

[3] S. ARGENTIERI "Introduction to image compression "Institute Of Robotics And Intelligent Systems Paris 6 France 2009.

[4] BENJAMIN HARBELOT YOAN "Project mathematical Fourier Transform" University of Burgundy France 2010.

[5] E. FAVIER. 'Image analysis and processing, principles of computer vision'’. ENISE France.

[6] AMAAR, E.M. SAAD, I. ASHOUR AND M. ELZORKANY Electronics Department, National Telecommunication Institute (NTI) Image "Compression Using K-Space Transformation Technique" Recent Researches in Communications, Electronics, Signal Processing and Automatic Control 2012.

[7] KUMAR, K.SINGH, R.KHANNA Dept. of ECE Thapar University, Patiala "Satellite Image Compression using Fractional Fourier Transform" International Journal of Computer Applications (0975 - 8887) Volume 50 - No.3, July 2012.

[8] MOULAY LAKHDAR, M. KANDOUCI, B. BELGHEIT "Image compression by wavelet transforms and adaptive vector quantization" University Of Bechar and Department of Electronic faculty of Science \& Engineering University Djilali Liabes. IMAGE conference Biskra Algeria 2009.

[9] E. FAVIER “image compression Part H” National School of engineers Saint-Etienne France 2008. 